Discrete Comput Geom 28:19-27 (2002)

DOI: $10.1007 / \mathrm{s} 00454-001-0079-y$

\title{
The Tilt Formula for Generalized Simplices in Hyperbolic Space
}

\author{
Akira Ushijima \\ Interactive Research Center of Science, Graduate School of Science and Engineering, \\ Tokyo Institute of Technology, Tokyo 152-8551, Japan \\ ushijima@math.titech.ac.jp
}

\begin{abstract}
For a simplex in Lorentzian space whose vertices are in the positive light cone, Weeks defined the "tilt" relative to each face. It gave us an efficient tool for deciding whether or not the dihedral angle between two simplices holding a face in common is convex. $\mathrm{He}$ also provided an efficient formula, called the "tilt formula," to obtain tilts from the intrinsic hyperbolic structure of the simplex when its dimension is two or three. Sakuma and Weeks generalized it to general dimensions. In this paper we generalize the concept of the tilt and the tilt formula to the case where not all vertices are in the positive light cone. A key to our generalization is to give a correspondence between points and hyperplanes (or half-spaces) in Lorentzian space.
\end{abstract}

\section{Introduction}

In [EP] Epstein and Penner gave a method for decomposing any noncompact complete cusped hyperbolic manifold of finite volume with weight at each cusp into ideal polyhedra. This decomposition is called the Euclidean decomposition, and defined via a convex hull construction in Lorentzian space. Each vertex of the hull is in the positive light cone and corresponds to a lift of a cusp, and each face of the hull corresponds to an ideal polyhedron in the manifold. Especially, if all weights are equal, then the decomposition is invariant under the action of its fundamental group and is called the canonical decomposition.

By its definition, the canonical decomposition of a cusped hyperbolic manifold is determined by its intrinsic hyperbolic structure. So this decomposition becomes a useful tool for studying such a manifold. When the dimension is two, this gives us a canonical cellular decomposition of the Teichmüller space of hyperbolic surfaces (see, for example, $[\mathrm{P}])$, and when the dimension is three, this is useful for the classification of hyperbolic manifolds, determination of their isometry group and so on (see, for example, [SW1]). So 
an efficient tool was required to decide whether or not a given polyhedral decomposition is canonical.

One of the earliest suggestions to this requirement was given by Penner in Proposition 2.6(b) of [P], which gave us a criterion of convexity when simplices are (twodimensional) ideal triangles. His idea was translated by Näätänen [N1, Lemma 3.3] into the case where simplices are triangles, and by Ushijima [U2, Proposition 3.5(2)] into the case where simplices are truncated triangles (i.e., orthogonal hexagons). These criteria are used for studying the Teichmüller space of such surfaces.

Independent of the above development, Weeks gave [W1, Proposition 3.1] a criterion of convexity when simplices are two- and three-dimensional ideal simplices. For a simplex in Lorentzian space whose vertices are in the positive light cone, he defined a real value called tilt on each of its faces. Using this value, we can easily decide whether or not the dihedral angle between two simplices holding a face in common is convex. Since the canonical decomposition is defined from a convex hull in Lorentzian space, it becomes a useful tool to determine whether or not a given polyhedral decomposition of the manifold is canonical in the previous sense. He also provided an efficient formula, called the tilt formula, to obtain tilts from the intrinsic hyperbolic structure of the simplex when its dimension is two (see Theorem 3.2 of [W1]) and three (see Theorem 5.1 of [W1]). He made a program, called "SnapPea," for creating and studying three-dimensional hyperbolic manifolds [W2]. In this program the formula is used for determining the canonical decomposition of a given three-dimensional hyperbolic manifold. SnapPea is so useful that it is used in many researches, especially in topology and astrophysics (see, for example, [CDW] and [I]). Later Sakuma and Weeks [SW2] generalized the tilt formula to general dimensions.

On the other hand, Kojima [K1], [K2] gave a method for decomposing any complete hyperbolic manifold of finite volume with nonempty totally geodesic boundary into partially truncated polyhedra. We note here that this decomposition is not a complete generalization of Epstein-Penner's one. However, this decomposition is also useful in studying such hyperbolic manifolds (see, for example, [U1] and [U2]), and convex hulls in Lorentzian space still play an important role. Therefore it is also meaningful to make an efficient tool for the decision of the canonical decomposition, namely, to generalize the tilt and the tilt formula. The purpose of the paper is to do so (see Definition 3.1 and Theorem 3.3).

This paper is organized as follows: in Section 2 we recall some basic facts about Lorentzian space and hyperbolic geometry. One important task of this section is to give a correspondence between points and hyperplanes (or half-spaces) in Lorentzian space. This correspondence is a generalization of the well-known duality between points in the hyperboloid of one sheet and half-spaces in hyperbolic space. In Section 3 we first define a generalized $n$-simplex in the projective model of the $n$-dimensional hyperbolic space. Roughly speaking, this is a partially truncated $n$-dimensional simplex of finite volume. Next we define the tilt of a generalized $n$-simplex relative to an internal face, and obtain a relationship between tilts and the convexity of two adjoining generalized $n$-simplices (see Proposition 3.2). Finally we state Theorem 3.3, which shows how to obtain tilts from the intrinsic hyperbolic structure of a generalized $n$-simplex, by imitating the method in [SW2]. 


\section{Lorentzian Space and Hyperbolic Geometry}

The $(n+1)$-dimensional Lorentzian space $\mathbb{E}^{1, n}$ is the real vector space $\mathbb{R}^{n+1}$ of dimension $n+1$ with the Lorentzian inner product $\langle\boldsymbol{x}, \boldsymbol{y}\rangle:=-x_{0} y_{0}+x_{1} y_{1}+\cdots+x_{n} y_{n}$, where $\boldsymbol{x}=\left(x_{0}, x_{1}, \ldots, x_{n}\right)$ and $\boldsymbol{y}=\left(y_{0}, y_{1}, \ldots, y_{n}\right)$. In this paper we assume $n \geq 2$. If $\sqrt{\langle\boldsymbol{x}, \boldsymbol{x}\rangle}$ is zero (resp. positive, imaginary), then $\boldsymbol{x}$ is said to be light-like (resp. space-like, timelike). The coordinate $x_{0}$ of $\mathbb{E}^{1, n}$ is called the height. We denote by $L^{+}$the set of light-like vectors with positive height; $L^{+}:=\left\{\boldsymbol{x} \in \mathbb{E}^{1, n} \mid\langle\boldsymbol{x}, \boldsymbol{x}\rangle=0\right.$ and $\left.x_{0}>0\right\}$.

Let $H_{T}^{+}:=\left\{\boldsymbol{x} \in \mathbb{E}^{1, n} \mid\langle\boldsymbol{x}, \boldsymbol{x}\rangle=-1\right.$ and $\left.x_{0}>0\right\}$ be the upper sheet of the (standard) hyperboloid of two sheets. The restriction of the quadratic form induced by $\langle\cdot, \cdot\rangle$ on $\mathbb{E}^{1, n}$ to the tangent space of $H_{T}^{+}$is positive definite and gives a Riemannian metric on $\mathrm{H}_{T}^{+}$. The space obtained from $H_{T}^{+}$equipped with the metric above is called the hyperboloid model of the $n$-dimensional hyperbolic space. If $\boldsymbol{x}$ and $\boldsymbol{y}$ are points in $H_{T}^{+}$and $d$ denotes the hyperbolic distance between $\boldsymbol{x}$ and $\boldsymbol{y}$, then the following relation holds (see, for example, p. 45 of [N2] or Proposition 2.4.5(a) of [T]): $\langle\boldsymbol{x}, \boldsymbol{y}\rangle=-\cosh d$. The (standard) hyperboloid of one sheet $H_{S}$ is defined to be $H_{S}:=\left\{\boldsymbol{x} \in \mathbb{E}^{1, n} \mid\langle\boldsymbol{x}, \boldsymbol{x}\rangle=1\right\}$.

We denote by $\mathcal{P}$ the radial projection from $\mathbb{E}^{1, n}-\left\{x \in \mathbb{E}^{1, n} \mid x_{0}=0\right\}$ to an affine hyperplane $\mathbf{P}_{1}^{n}:=\left\{\boldsymbol{x} \in \mathbb{E}^{1, n} \mid x_{0}=1\right\}$ along the ray from the origin $\boldsymbol{o}=(0,0, \ldots, 0)$. The projection $\mathcal{P}$ is a homeomorphism on $H_{T}^{+}$to the $n$-dimensional open unit ball $\mathbf{B}^{n}$ in $\mathbf{P}_{1}^{n}$ centered at $(1,0,0, \ldots, 0)$. We call $\mathbf{B}^{n}$ with the metric induced by the projection the projective model of the $n$-dimensional hyperbolic space. This projection also induces the mapping from $\mathbb{E}^{1, n}-\{\boldsymbol{o}\}$ to the $n$-dimensional real projective space $\mathbf{P}^{n}=\mathbf{P}_{1}^{n} \cup \mathbf{P}_{\infty}^{n}$, where $\mathbf{P}_{\infty}^{n}$ is the set of lines in the affine hyperplane $\left\{\boldsymbol{x} \in \mathbb{E}^{1, n} \mid x_{0}=0\right\}$ through $\boldsymbol{o}$ and form the sphere at infinity. Now we use the notation $\mathcal{P}$ for the mapping obtained as above for brevity since there will be no confusion.

A vector subspace of $\mathbb{E}^{1, n}$ is said to be time-like if it has a time-like vector, space-like if every nonzero vector in it is space-like, or light-like otherwise. Suppose $P$ is a timelike linear hyperplane, and let $R$ be a half-space in $\mathbb{E}^{1, n}$ bounded by $P$. Then we can associate a unique vector $\boldsymbol{w} \in H_{S}$ so that $\langle\boldsymbol{w}, \boldsymbol{q}\rangle \leq 0$ for any $\boldsymbol{q} \in R$. This establishes a well-known duality between points on $H_{S}$ and half-spaces in $\mathbb{E}^{1, n}$ bounded by time-like linear hyperplanes. Now we give a generalization of this duality. For an arbitrary vector $\boldsymbol{u}$ in $\mathbb{E}^{1, n}$, we define a half-space $R_{\boldsymbol{u}}$ and a hyperplane $P_{\boldsymbol{u}}$ in $\mathbb{E}^{1, n}$ as follows:

$$
\begin{aligned}
R_{\boldsymbol{u}} & :=\left\{\boldsymbol{x} \in \mathbb{E}^{1, n} \mid\langle\boldsymbol{x}, \boldsymbol{u}\rangle \leq \frac{\langle\boldsymbol{u}, \boldsymbol{u}\rangle-1}{2}\right\}, \\
P_{\boldsymbol{u}} & :=\left\{\boldsymbol{x} \in \mathbb{E}^{1, n} \mid\langle\boldsymbol{x}, \boldsymbol{u}\rangle=\frac{\langle\boldsymbol{u}, \boldsymbol{u}\rangle-1}{2}\right\}=\partial R_{\boldsymbol{u}} .
\end{aligned}
$$

We denote by $\Gamma_{\boldsymbol{u}}\left(\right.$ resp. $\Pi_{\boldsymbol{u}}$ ) the intersection of $R_{\boldsymbol{u}}$ (resp. $P_{\boldsymbol{u}}$ ) and $H_{T}^{+}$. We call $\boldsymbol{u}$ a normal vector to $P_{u}\left(\right.$ or $\left.\Pi_{u}\right)$. Then we can easily obtain the following proposition:

\section{Proposition 2.1.}

(1) If $\boldsymbol{u} \in H_{T}^{+}$, then $\Pi_{\boldsymbol{u}}=\{\boldsymbol{u}\}$.

(2) If $\boldsymbol{u} \in L^{+}$, then $\Pi_{\boldsymbol{u}}$ is a horosphere whose center is a line directed by $\boldsymbol{u}$.

(3) If $\boldsymbol{u} \in H_{S}$, then $\Pi_{\boldsymbol{u}}$ is a geodesic hyperplane. 
Remark 2.2. More generally, if $\langle\boldsymbol{u}, \boldsymbol{u}\rangle<0$ and $u_{0}>0$, then $\Pi_{\boldsymbol{u}}$ is a sphere centered at $n(\boldsymbol{u}):=\boldsymbol{x} / \sqrt{|\langle\boldsymbol{x}, \boldsymbol{x}\rangle|}$, and if $\langle\boldsymbol{u}, \boldsymbol{u}\rangle>0$, then $\Pi_{\boldsymbol{u}}$ is an equidistant hypersurface whose axial hyperplane is $\Pi_{n(\boldsymbol{u})}$.

Furthermore, if the other cases hold, i.e., if $\langle\boldsymbol{u}, \boldsymbol{u}\rangle \leq 0$ and $u_{0} \leq 0$, then $\Pi_{\boldsymbol{u}}=$ $P_{u} \cap H_{T}^{+}=\emptyset$. This means that almost every point in $\mathbb{E}^{1, n}$ can be given an appropriate geometric meaning as the correspondence to all fundamental geometric objects in the hyperbolic space.

Now we define the generalized distance between two geometric objects. Here the word "generalized" means that the distance defined below is a unification of distances (in the usual sense) and the angles.

Definition 2.3 (Generalized Distance). For a point $\boldsymbol{x}$ in $H_{S}$ and $\boldsymbol{y}$ in $\left(H_{T}^{+} \cup L^{+} \cup H_{S}\right) \cap$ $R_{x}$, the generalized distance $d$ between $\Pi_{x}$ and $\Pi_{y}$ is defined as follows:

Case 1. If $\boldsymbol{y} \in H_{T}^{+} \cap R_{x}$, then $d$ is the distance (in the usual sense) between $\Pi_{x}$ and $\Pi_{y}=\{\boldsymbol{y}\}$.

Case 2. If $y \in L^{+} \cap R_{x}$, then $d$ is the signed distance (defined below) between $\Pi_{x}$ and $\Pi_{y}$.

Case 3. If $\boldsymbol{y} \in H_{S} \cap R_{\boldsymbol{x}}$, then $(\langle\boldsymbol{x}, \boldsymbol{y}\rangle \leq 0)$ and we have two different cases:

Case 3.1. If $-1<\langle\boldsymbol{x}, \boldsymbol{y}\rangle(\leq 0)$, i.e., if $\Pi_{x}$ and $\Pi_{\boldsymbol{y}}$ intersect each other, then $d:=$ $\sqrt{-1} \theta$, where $\theta$ is the dihedral angle between $\Pi_{x}$ and $\Pi_{y}$ measured in $\Gamma_{x} \cap \Gamma_{y}$.

Case 3.2. If $\langle\boldsymbol{x}, \boldsymbol{y}\rangle \leq-1$, i.e., if $\Pi_{x}$ and $\Pi_{y}$ are parallel or ultraparallel, then $d$ is the distance (in the usual sense) between $\Pi_{x}$ and $\Pi_{y}$.

Here the signed distance between a geodesic hyperplane $\Pi_{x}$ and a horosphere $\Pi_{y}$ is the signed distance between $\Pi_{x}$ and $z$, where $z$ is the intersecting point of $\Pi_{y}$ and the time-like linear plane spanned by $\boldsymbol{x}$ and $\boldsymbol{y}$ (if $\boldsymbol{y} \notin P_{x}$ ), and the sign is defined to be positive (resp. negative) if $z \in \Gamma_{x}$ (resp. $z \notin \Gamma_{x}$ ), that is, if $\langle\boldsymbol{x}, z\rangle \leq 0$ (resp. $\langle\boldsymbol{x}, z\rangle>0$ ). If $\boldsymbol{y} \in P_{x}$, then we define the signed distance as $+\infty$.

Summarizing several basic facts on hyperbolic geometry (see, for example, Theorems 3.2.8, 6.2.10, and 3.2.12 of [R]), we can obtain the following proposition:

Proposition 2.4. Let $\boldsymbol{x}$ be a point in $H_{S}$. For an arbitrary point $\boldsymbol{y} \in\left(H_{T}^{+} \cup L^{+} \cup H_{S}\right) \cap R_{\boldsymbol{x}}$, the following equality holds:

$$
\langle\boldsymbol{x}, \boldsymbol{y}\rangle=-\frac{e^{d}+v e^{-d}}{2}
$$

where $v:=\langle\boldsymbol{y}, \boldsymbol{y}\rangle$ and $d$ is the generalized distance between $\Pi_{x}$ and $\Pi_{y}$.

\section{Definitions of Generalized $\boldsymbol{n}$-Simplices and Tilts}

\subsection{The Definition of Generalized $n$-Simplices}

Let $U=\left\{\boldsymbol{u}_{0}, \boldsymbol{u}_{1}, \ldots, \boldsymbol{u}_{n}\right\}$ be a set of independent points in $H_{T}^{+} \cup L^{+} \cup H_{S}$. We divide $U$ into two disjoint subsets, say $U_{\text {in }}$ and $U_{\text {ex }}$ as follows: $U_{\text {in }}:=\left\{\boldsymbol{u} \in U \mid \boldsymbol{u} \in H_{T}^{+} \cup L^{+}\right\}$ 


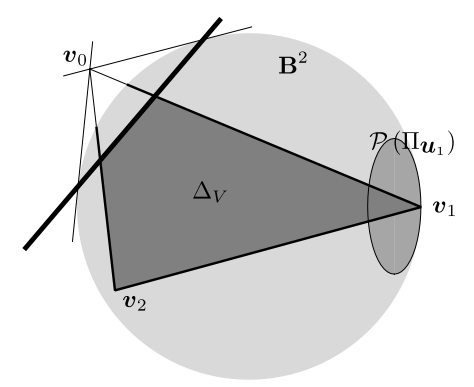

Fig. 1. An example of a generalized 2-simplex in $\mathbf{B}^{2}$.

and $U_{\text {ex }}:=\left\{\boldsymbol{u} \in U \mid \boldsymbol{u} \in H_{S}\right\}=U-U_{\text {in }}$. Without loss of generality, we may assume $U_{\text {ex }}=\left\{\boldsymbol{u}_{0}, \boldsymbol{u}_{1}, \ldots, \boldsymbol{u}_{k}\right\}$ and $U_{\text {in }}=\left\{\boldsymbol{u}_{k+1}, \boldsymbol{u}_{k+2}, \ldots, \boldsymbol{u}_{n}\right\}$ for some $k$ by changing indices if necessary. For any $i \in\{0,1, \ldots, n\}$, we denote by $F_{i}$ the linear hyperplane spanned by $\left\{\boldsymbol{u}_{0}, \boldsymbol{u}_{1}, \ldots, \boldsymbol{u}_{i-1}, \boldsymbol{u}_{i+1}, \ldots, \boldsymbol{u}_{n}\right\}$. Let $\triangle_{U}$ be the $n$-simplex in $\mathbb{E}^{1, n}$ with the vertex set $U$.

Now we suppose $U$ satisfies the following two conditions:

Condition 1. For any $i \in\{0,1, \ldots, k\}, R_{\boldsymbol{u}_{i}}-P_{\boldsymbol{u}_{i}} \supset\left\{\boldsymbol{u}_{0}, \boldsymbol{u}_{1}, \ldots, \boldsymbol{u}_{i-1}, \boldsymbol{u}_{i+1}, \ldots, \boldsymbol{u}_{n}\right\}$.

Condition 2. For any $i \in\{0,1, \ldots, n\}, F_{i} \cap H_{T}^{+} \neq \emptyset$.

Let $V$ be $\mathcal{P}(U)$ and let $\boldsymbol{v}_{i}$ be $\mathcal{P}\left(\boldsymbol{u}_{i}\right)$. Under these conditions, we define a generalized $n$-simplex $\Delta_{V}$ in $\mathbf{B}^{n}$ with vertex set $V$ as $\mathcal{P}\left(\triangle_{U} \cap \bigcap_{i=0}^{k} R_{u_{i}}\right)$ (see Fig. 1).

We regard the $\boldsymbol{v}_{i}$ 's as vertices of $\Delta_{V}$. Each face of $\Delta_{V}$ is either contained in $P_{\boldsymbol{u}_{i}}$ for some $i \in\{0,1, \ldots, k\}$ or in $F_{j}$ for some $j \in\{0,1, \ldots, n\}$. According to Kojima [K1], [K2], we call the former an external face of $\Delta_{V}$, and the later an internal face of $\Delta_{V}$ (especially, this internal face is the opposite face of $\boldsymbol{v}_{j}$ ). Now Condition 1 means that $\Pi_{\boldsymbol{u}_{i}}$ cuts off nothing but $\boldsymbol{v}_{i}$ from $V$, and Condition 2 means that for each vertex $\boldsymbol{v}_{i}$ its opposite (internal) face exists.

For a generalized $n$-simplex $\Delta_{V}$ with weight at all ideal vertices (here a "weight" is a real number), there exists a unique $n$-simplex $\triangle_{U}$ in $\mathbb{E}^{1, n}$ whose projection corresponds to $\Delta_{V}$. We note here that the correspondence between an ideal vertex $v$ of $\Delta_{V}$ with weight $w$ and its preimage $\boldsymbol{u}$ is defined as follows: $\mathcal{P}(\boldsymbol{u})=\boldsymbol{v}$ and $w=-\log \left(u_{0} \sqrt{2}\right)$, where $u_{0}$ is a height of $\boldsymbol{u}$. From now on we use the term "generalized $n$-simplex" as the simplex itself with weights at all ideal vertices. We call $\Delta_{U}$ the lift of a generalized $n$-simplex $\Delta_{V}$ in $\mathbf{B}^{n}, U$ the lift of the vertex set $V$, and $\boldsymbol{u}$ the lift of the vertex $\mathcal{P}(\boldsymbol{u}) \in V$.

\subsection{The Definition of Tilt and the Tilt Proposition}

Fix a generalized $n$-simplex $\Delta_{V}$ in $\mathbf{B}^{n}$, and take one of its internal faces $\Phi_{i}$. Then there is a unique point $\boldsymbol{m}_{i}$ in $H_{S}$ such that $\Phi_{i} \subset P_{\boldsymbol{m}_{i}}$ and $\Delta_{V} \subset R_{\boldsymbol{m}_{i}}$. We define the normal vector $\boldsymbol{p}$ to the lift $\triangle_{U}$ of $\Delta_{V}$ by the condition that $\langle\boldsymbol{p}, \boldsymbol{x}\rangle=-1$ for all $\boldsymbol{x} \in \triangle_{U}$. 
Definition 3.1. The tilt $t_{i}$ of $\Delta_{V}$ relative to $\Phi_{i}$ is defined as follows:

$$
t_{i}:=\left\langle\boldsymbol{m}_{i}, \boldsymbol{p}\right\rangle
$$

Proposition 3.2 following, corresponding to Proposition 3.1 of [W1], states how to use the tilt for deciding the convexity.

Proposition 3.2 (Tilt Proposition). Let $\triangle_{U_{0}}$ and $\triangle_{U_{1}}$ be two adjacent $n$-simplices in $\mathbb{E}^{1, n}$ that project to generalized $n$-simplices in $\mathbf{B}^{n}$. We denote by $t_{0}$ (resp. $t_{1}$ ) the tilt of $\Delta_{V_{0}}$ (resp. $\Delta_{V_{1}}$ ) relative to the joint face. Then the dihedral angle formed by $\triangle_{U_{0}}$ and $\Delta_{U_{1}}$ is convex (flat, concave, respectively) if and only if $t_{0}+t_{1}<0(=0,>0$, respectively).

Proof. Let $U_{0}=\left\{\boldsymbol{u}_{0}, \boldsymbol{u}_{1}, \ldots, \boldsymbol{u}_{n}\right\}$ and $U_{1}=\left\{\boldsymbol{u}_{1}, \boldsymbol{u}_{2}, \ldots, \boldsymbol{u}_{n+1}\right\}$. We denote by $\boldsymbol{m}$ the normal vector to the hyperplane containing the joint face satisfying that $\triangle_{U_{0}} \subset R_{m}$. Then, for an arbitrary $i \in\{1,2, \ldots, n\}$, we have $\left\langle\boldsymbol{u}_{i}, \boldsymbol{m}\right\rangle=0$. Furthermore, we have $\left\langle\boldsymbol{u}_{0}, \boldsymbol{m}\right\rangle<0$ and $\left\langle\boldsymbol{u}_{n+1}, \boldsymbol{m}\right\rangle>0$.

Since vectors $\boldsymbol{m}, \boldsymbol{u}_{1}, \boldsymbol{u}_{2}, \ldots, \boldsymbol{u}_{n}$ form a basis of $\mathbb{E}^{1, n}$, there exist unique real numbers $\alpha_{0}, \alpha_{1}, \ldots, \alpha_{n}$ such that

$$
\boldsymbol{u}_{0}=\alpha_{0} \boldsymbol{m}+\alpha_{1} \boldsymbol{u}_{1}+\alpha_{2} \boldsymbol{u}_{2}+\cdots+\alpha_{n} \boldsymbol{u}_{n}
$$

Similarly we have

$$
\boldsymbol{u}_{n+1}=\alpha_{0}^{\prime} \boldsymbol{m}+\alpha_{1}^{\prime} \boldsymbol{u}_{1}+\alpha_{2}^{\prime} \boldsymbol{u}_{2}+\cdots+\alpha_{n}^{\prime} \boldsymbol{u}_{n}
$$

for some $\alpha_{0}^{\prime}, \alpha_{1}^{\prime}, \ldots, \alpha_{n}^{\prime} \in \mathbb{R}$. We note that $\alpha_{0}^{\prime}>0$, since $\left\langle\boldsymbol{u}_{n+1}, \boldsymbol{m}\right\rangle>0$.

Let $\boldsymbol{p}_{0}$ (resp. $\left.\boldsymbol{p}_{1}\right)$ be the normal vector to $\triangle_{U_{0}}$ (resp. $\triangle_{U_{1}}$ ). Now $\boldsymbol{p}_{0}$ is also expressed as a linear combination of $\boldsymbol{m}, \boldsymbol{u}_{1}, \boldsymbol{u}_{2}, \ldots, \boldsymbol{u}_{n}$ as follows:

$$
\boldsymbol{p}_{0}=\beta_{0} \boldsymbol{m}+\beta_{1} \boldsymbol{u}_{1}+\beta_{2} \boldsymbol{u}_{2}+\cdots+\beta_{n} \boldsymbol{u}_{n},
$$

where $\beta_{0}, \beta_{1}, \ldots, \beta_{n} \in \mathbb{R}$. Then we have the following equation:

$$
\left(\begin{array}{c}
\left\langle\boldsymbol{p}_{0}, \boldsymbol{u}_{1}\right\rangle \\
\left\langle\boldsymbol{p}_{0}, \boldsymbol{u}_{2}\right\rangle \\
\vdots \\
\left\langle\boldsymbol{p}_{0}, \boldsymbol{u}_{n}\right\rangle
\end{array}\right)=\left(\begin{array}{cccc}
\left\langle\boldsymbol{u}_{1}, \boldsymbol{u}_{1}\right\rangle & \left\langle\boldsymbol{u}_{1}, \boldsymbol{u}_{2}\right\rangle & \cdots & \left\langle\boldsymbol{u}_{1}, \boldsymbol{u}_{n}\right\rangle \\
\left\langle\boldsymbol{u}_{2}, \boldsymbol{u}_{1}\right\rangle & \left\langle\boldsymbol{u}_{2}, \boldsymbol{u}_{2}\right\rangle & \cdots & \left\langle\boldsymbol{u}_{2}, \boldsymbol{u}_{n}\right\rangle \\
\vdots & \vdots & \ddots & \vdots \\
\left\langle\boldsymbol{u}_{n}, \boldsymbol{u}_{1}\right\rangle & \left\langle\boldsymbol{u}_{n}, \boldsymbol{u}_{2}\right\rangle & \cdots & \left\langle\boldsymbol{u}_{n}, \boldsymbol{u}_{n}\right\rangle
\end{array}\right),\left(\begin{array}{c}
\beta_{1} \\
\beta_{2} \\
\vdots \\
\beta_{n}
\end{array}\right)=\left(\begin{array}{c}
-1 \\
-1 \\
\vdots \\
-1
\end{array}\right)
$$

that is,

$$
\sum_{i=1}^{n} \beta_{i}\left\langle\boldsymbol{u}_{j}, \boldsymbol{u}_{i}\right\rangle=-1 \quad \text { for } \quad j \in\{1,2, \ldots, n\} .
$$

Similarly we have

$$
\boldsymbol{p}_{1}=\beta_{0}^{\prime} \boldsymbol{m}+\beta_{1}^{\prime} \boldsymbol{u}_{1}+\beta_{2}^{\prime} \boldsymbol{u}_{2}+\cdots+\beta_{n}^{\prime} \boldsymbol{u}_{n}
$$


for some $\beta_{0}^{\prime}, \beta_{1}^{\prime}, \ldots, \beta_{n}^{\prime} \in \mathbb{R}$. Then, in the same fashion as (3.1), we have

$$
\sum_{i=1}^{n} \beta_{i}^{\prime}\left\langle\boldsymbol{u}_{j}, \boldsymbol{u}_{i}\right\rangle=-1 \quad \text { for } \quad j \in\{1,2, \ldots, n\}
$$

Using the relations above, we can compute $\left\langle\boldsymbol{p}_{1}, \boldsymbol{u}_{n+1}\right\rangle$ as follows:

$$
\begin{aligned}
-1=\left\langle\boldsymbol{p}_{1}, \boldsymbol{u}_{n+1}\right\rangle= & \alpha_{0}^{\prime} \beta_{0}^{\prime}+\alpha_{1}^{\prime}\left(\beta_{1}^{\prime}\left\langle\boldsymbol{u}_{1}, \boldsymbol{u}_{1}\right\rangle+\beta_{2}^{\prime}\left\langle\boldsymbol{u}_{1}, \boldsymbol{u}_{2}\right\rangle+\cdots+\beta_{n}^{\prime}\left\langle\boldsymbol{u}_{1}, \boldsymbol{u}_{n}\right\rangle\right) \\
& +\alpha_{2}^{\prime}\left(\beta_{1}^{\prime}\left\langle\boldsymbol{u}_{2}, \boldsymbol{u}_{1}\right\rangle+\beta_{2}^{\prime}\left\langle\boldsymbol{u}_{2}, \boldsymbol{u}_{2}\right\rangle+\cdots+\beta_{n}^{\prime}\left\langle\boldsymbol{u}_{2}, \boldsymbol{u}_{n}\right\rangle\right) \\
& +\cdots \\
& +\alpha_{n}^{\prime}\left(\beta_{1}^{\prime}\left\langle\boldsymbol{u}_{n}, \boldsymbol{u}_{1}\right\rangle+\beta_{2}^{\prime}\left\langle\boldsymbol{u}_{n}, \boldsymbol{u}_{2}\right\rangle+\cdots+\beta_{n}^{\prime}\left\langle\boldsymbol{u}_{n}, \boldsymbol{u}_{n}\right\rangle\right) \\
= & \alpha_{0}^{\prime} \beta_{0}^{\prime}-\sum_{i=1}^{n} \alpha_{i}^{\prime} .
\end{aligned}
$$

Thus we have the following relation:

$$
\sum_{i=1}^{n} \alpha_{i}^{\prime}=\alpha_{0}^{\prime} \beta_{0}^{\prime}+1
$$

The hyperplane $P_{i}$, where $i=1$ or 2 , is defined to be $P_{i}:=\left\{\boldsymbol{x} \in \mathbb{E}^{1, n} \mid\left\langle\boldsymbol{x}, \boldsymbol{p}_{i}\right\rangle=-1\right\}$. Then $P_{i}$ contains $\triangle_{U_{i}}$. Now the dihedral angle formed by $\triangle_{U_{0}}$ and $\triangle_{U_{1}}$ is convex if and only if $P_{0}$ separates $\boldsymbol{u}_{n+1}$ from the origin $\boldsymbol{o}$, or, equivalently, if and only if $P_{1}$ separates $\boldsymbol{u}_{0}$ from $\boldsymbol{o}$. This condition is equivalent to the one when $\left\langle\boldsymbol{u}_{i}, \boldsymbol{p}_{j}\right\rangle<-1$, where $i, j)=(n+1,0)$ or $(0,1)$. So using (3.1) and (3.2), we can compute $\left\langle\boldsymbol{u}_{n+1}, \boldsymbol{p}_{0}\right\rangle$ as follows:

$$
\begin{aligned}
-1>\left\langle\boldsymbol{u}_{n+1}, \boldsymbol{p}_{0}\right\rangle= & \alpha_{0}^{\prime} \beta_{0}+\alpha_{1}^{\prime}\left(\beta_{1}\left\langle\boldsymbol{u}_{1}, \boldsymbol{u}_{1}\right\rangle+\beta_{2}\left\langle\boldsymbol{u}_{1}, \boldsymbol{u}_{2}\right\rangle+\cdots+\beta_{n}\left\langle\boldsymbol{u}_{1}, \boldsymbol{u}_{n}\right\rangle\right) \\
& +\alpha_{2}^{\prime}\left(\beta_{1}\left\langle\boldsymbol{u}_{2}, \boldsymbol{u}_{1}\right\rangle+\beta_{2}\left\langle\boldsymbol{u}_{2}, \boldsymbol{u}_{2}\right\rangle+\cdots+\beta_{n}\left\langle\boldsymbol{u}_{2}, \boldsymbol{u}_{n}\right\rangle\right) \\
& +\cdots \\
& +\alpha_{n}^{\prime}\left(\beta_{1}\left\langle\boldsymbol{u}_{n}, \boldsymbol{u}_{1}\right\rangle+\beta_{2}\left\langle\boldsymbol{u}_{n}, \boldsymbol{u}_{2}\right\rangle+\cdots+\beta_{n}\left\langle\boldsymbol{u}_{n}, \boldsymbol{u}_{n}\right\rangle\right) \\
= & \alpha_{0}^{\prime} \beta_{0}-\sum_{i=1}^{n} \alpha_{i}^{\prime} \\
= & \alpha_{0}^{\prime} \beta_{0}-\left(\alpha_{0}^{\prime} \beta_{0}^{\prime}+1\right) .
\end{aligned}
$$

Thus we obtain $\beta_{0}-\beta_{0}^{\prime}<0$, since $\alpha_{0}^{\prime}>0$. Furthermore, $t_{0}=\left\langle\boldsymbol{m}, \boldsymbol{p}_{0}\right\rangle=\beta_{0}$ and $t_{1}=\left\langle-\boldsymbol{m}, \boldsymbol{p}_{1}\right\rangle=-\beta_{0}^{\prime}$, and we have $t_{0}+t_{1}<0$ if and only if the dihedral angle formed by $\triangle_{U_{0}}$ and $\triangle_{U_{1}}$ is convex. The proofs of the other cases are analogous. We have thus proved Proposition 3.2.

\subsection{The Tilt Formula}

The definition of the canonical decomposition requires that a tilt is obtained from the intrinsic hyperbolic structure of a generalized $n$-simplex, and the following tilt formula shows how to do it. 


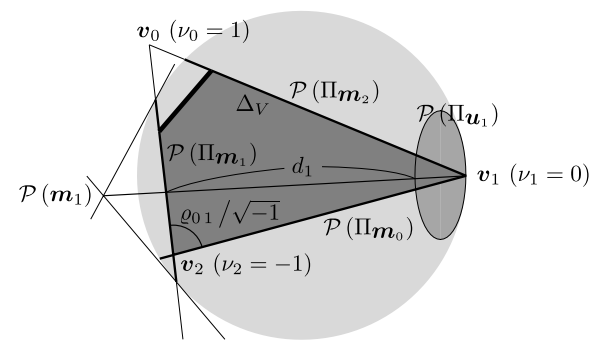

Fig. 2. Values for getting tilts.

Fix a generalized $n$-simplex $\Delta_{V}$ in $\mathbf{B}^{n}$, and denote by $U=\left\{\boldsymbol{u}_{0}, \boldsymbol{u}_{1}, \ldots, \boldsymbol{u}_{n}\right\}$ the lift of $V$. Let $v_{i}:=\left\langle\boldsymbol{u}_{i}, \boldsymbol{u}_{i}\right\rangle$ and let $d_{i}$ be the generalized distance between $\Pi_{\boldsymbol{u}_{i}}$ and $\Pi_{\boldsymbol{m}_{i}}$, where $\boldsymbol{m}_{i}$ is a point in $H_{S}$ with $\Phi_{i} \subset P_{\boldsymbol{m}_{i}}$ and $\Delta_{V} \subset R_{\boldsymbol{m}_{i}}$. Then we define $Q_{i}:=2 /\left(e^{d_{i}}+v_{i} e^{-d_{i}}\right)$. We denote by $\varrho_{i j}$ the generalized distance between $\Pi_{m_{i}}$ and $\Pi_{\boldsymbol{m}_{j}}$. We note here that we can obtain $v_{i}, d_{i}$ and $\varrho_{i j}$ only from the intrinsic hyperbolic structure of $\Delta_{V}$ (see Fig. 2). Then we have Theorem 3.3 following, a generalization of Theorem 2.1 of [SW2]:

Theorem 3.3 (Tilt Formula). The tilt of a generalized $n$-simplex relative to each of its (codimension one) internal faces may be computed as follows:

$$
\left(\begin{array}{c}
t_{0} \\
t_{1} \\
t_{2} \\
\vdots \\
t_{n}
\end{array}\right)=\left(\begin{array}{ccccc}
1 & -\cosh \varrho_{01} & -\cosh \varrho_{02} & \cdots & -\cosh \varrho_{0 n} \\
-\cosh \varrho_{10} & 1 & -\cosh \varrho_{12} & \cdots & -\cosh \varrho_{1 n} \\
-\cosh \varrho_{20} & -\cosh \varrho_{21} & 1 & \cdots & -\cosh \varrho_{2 n} \\
\vdots & \vdots & \vdots & \ddots & \vdots \\
-\cosh \varrho_{n 0} & -\cosh \varrho_{n 1} & -\cosh \varrho_{n 2} & \cdots & 1
\end{array}\right)\left(\begin{array}{c}
Q_{0} \\
Q_{1} \\
Q_{2} \\
\vdots \\
Q_{n}
\end{array}\right) .
$$

We call the $(n+1) \times(n+1)$ matrix on the right side of the above formula the Gram matrix of the generalized n-simplex $\Delta_{V}$ (see [V]). The proof of this theorem is nearly a word-by-word translation of that of Theorem 2.1 of [SW2] together with replacing Lemma 2.4 of [SW2] by Proposition 2.4 in this paper. Thus we omit the proof.

\section{Acknowledgments}

The author thanks Professor Katsuo Kawakubo for his encouragement. The author also expresses his sincere gratitude to Professor Makoto Sakuma and Doctor Jeffrey R. Weeks for their helpful comments and advice. His thanks go also to the referee for his/her careful reading. 


\section{References}

[CDW] P. J. Callahan, J. C. Dean and J. R. Weeks, The simplest hyperbolic knots, J. Knot Theory Ramifications 8 (1999), 279-297.

[EP] D. B. A. Epstein and R. C. Penner, Euclidean decompositions of noncompact hyperbolic manifolds, J. Differential Geom. 27 (1988), 67-80.

[I] K. T. Inoue, Geometric Gaussianity and non-Gaussianity in the cosmic microwave background, Phys. Rev. D 62 (2000), 103001-1-103001-16.

[K1] S. Kojima, Polyhedral decomposition of hyperbolic manifolds with boundary, in On the Geometric Structure of Manifolds, Proceedings of Workshops in Pure Mathematics, Vol. 10, The Korean Academic Council, Korea, 1990, pp. 37-57.

[K2] S. Kojima, Polyhedral decomposition of hyperbolic 3-manifolds with totally geodesic boundary, in Aspects of Low Dimensional Manifolds, Advanced Studies in Pure Mathematics, Vol. 20, Kinokuniya, Tokyo, 1992, pp. 93-112.

[N1] M. Näätänen, A cellular parameterization for closed surfaces with a distinguished point, Ann. Acad. Sci. Fenn. Ser. A I Math. 18 (1993), 45-64.

[N2] M. Nakaoka, Soukyoku Kikagaku Nyuumon (Introduction to Hyperbolic Geometry) (in Japanese), Library of Mathematical Sciences, Vol. 5, Saiensu-sya, Tokyo, 1993.

[P] R. C. Penner, The decorated Teichmüller space of punctured surfaces, Comm. Math. Phys. 113 (1987), 299-339.

[R] J. G. Ratcliffe, Foundations of Hyperbolic Manifolds, Graduate Texts of Mathematics, Vol. 149, Springer-Verlag, New York, 1994.

[SW1] M. Sakuma and J. R. Weeks, Examples of canonical decompositions of hyperbolic link complements, Japan. J. Math. 21 (1995), 393-439.

[SW2] M. Sakuma and J. R. Weeks, The generalized tilt formula, Geom. Dedicata 55 (1995), 115-123.

[T] W. P. Thurston, Three-Dimensional Geometry and Topology, Princeton Mathematical Series, Vol. 35, Princeton University Press, Princeton, NJ, 1997.

[U1] A. Ushijima, The canonical decompositions of some family of compact orientable hyperbolic 3manifolds with totally geodesic boundary, Geom. Dedicata 78 (1999), 21-47.

[U2] A. Ushijima, A canonical cellular decomposition of the Teichmüller space of compact surfaces with boundary, Comm. Math. Phys. 201 (1999), 305-326.

[V] È. B. Vinberg, Discrete groups generated by reflections in Lobačevskiǔ spaces, Math. USSR-Sb. 1 (1967), 429-444.

[W1] J. R. Weeks, Convex hulls and isometries of cusped hyperbolic 3-manifolds, Topology Appl.52 (1993), $127-149$.

[W2] J. R. Weeks, SnapPea, A program for creating and studying hyperbolic 3-manifolds, available at http://www. northnet.org/weeks/index/SnapPea.html.

Received May 7, 2001, and in revised form November 13, 2001. Online publication April 19, 2002. 